\title{
Evolution of the Pancaking Effect in a $\Lambda$ CDM Cosmology
}

\author{
Yookyung Noh and Jounghun Lee \\ Department of Physics and Astronomy, FPRD, Seoul National University, Seoul 151-747, \\ Korea \\ ykyung@astro.snu.ac.kr, jounghun@astro.snu.ac.kr
}

\begin{abstract}
We explore the evolution of the large-scale anisotropy in the velocity field caused by the gravitational pancaking effect assuming a $\Lambda$ CDM universe. The Millennium Run halo catalogs at four different redshifts, $z=0,0.5,1$ and $z=2$ are analyzed to find that the pancaking effect starts to intervene the hierarchical structure formation at redshift $z=2$ when a characteristic pancake scale is around $3 h^{-1} \mathrm{Mpc}$. It is also clearly shown how the degree and scale of the pancaking effect changes with time. An analytic model based on the Zel'dovich approximation is presented to explain quantitatively the evolution of the velocitypancake alignment. A cosmological implication of our finding and a possibility of detecting a signal in real universe are discussed.
\end{abstract}

Subject headings: cosmology: theory — large-scale structure of universe

\section{INTRODUCTION}

The observed filamentary distribution of the large scale structure in the universe, which are often called the cosmic web, have so far motivated plenty of works. After the ingenious breakthrough made by Bond, Kofman, \& Pogosyan (1996), a theoretical framework has been provided within which the characteristic properties of the cosmic web can be explained qualitatively in terms of the tidal influences in the universe dominated by the cold dark matter (CDM).

The influence of the tidal forces on the orientations and distributions of the large scale structure has been a target of many fruitful studies which either numerically or observationally proved that the tidal effect is strongest near the pancakes.

A pancake refers to the two-dimensional sheet-like structure in the universe, which form through gravitational collapse along the local tidal field. The first order Lagrangian 
perturbation theory, the Zel'dovich approximation (Zel'dovich 1970), is probably the most popular model for the pancake formation which was originally suggested assuming the hot dark matter (HDM) dominated universe. The cosmic web theory, however, showed brilliantly that the presence of pancakes are also very possible in the CDM-dominated universe due to the large scale coherence of the tidal field. N-body simulations of CDM particles indeed demonstrated that the first collapsed objects look like pancakes (Shandarin et al. 1995).

The large-scale coherence of the tidal field that is responsible for the formation of pancakes in turn causes the dark matter halos near or on the pancakes to have strong spatial correlations in the orientations (Aragon-Calvo et al. 2007; Brunino et al. 2007; Hahn et al. 2007). The reason that the degree of the tidally induced alignments is highest near the pancakes can be understood given the fact that the pancakes are the first collapsed objects, being still in linear regime while the filaments are more severely modified by the nonlinear process.

The formation of pancakes induces not only the spatial correlations in the halo orientations but also the anisotropy in the velocity field. In our previous paper (Noh and Lee 2006, hereafter, NL06), we have for the first time detected a $5 \sigma$ significant signal of the velocity anisotropy induced by the pancaking effect in the high-resolution N-body simulation. In spite that the signal is found to be very weak because of the non-conserved nature of the velocity field, the velocity anisotropy holds a crucial key to understanding the pancaking effect on the structure formation.

In the light of the first detection of NL06, a couple of questions naturally arise: When does the pancaking effect influence most dominantly the formation of dark halos?; How does the pancake scale change with time?; What are the implications and consequences of the pancaking effect on the structure formation?; Is the standard theory capable of quantifying the velocity anisotropy?. Our goal here is to provide answers to these questions.

We organize this paper as follows. In $\S 2$, we introduce the analyzing method to investigate the evolution of the pancaking effect in the Millennium Simulation. We describe analytic modeling and compare it with the results obtained in the simulation catalog in $\S 3$. Finally, we discuss and conclude the results.

\section{SIGNALS FROM SIMULATION}

To investigate the evolution of local pancaking effect, we use the Millennium Run simulation halo catalogs for the concordance $\Lambda$ CDM cosmology (Springel et al 2005). A periodic box of this simulation is $500 h^{-1} \mathrm{Mpc}$ on each side and the cosmological parameters are 
$\Omega_{m}=0.25, \Omega_{\Lambda}=1-\Omega_{m}, h=0.73$, and $\sigma_{8}=0.9$. We choose four different redshift cata$\operatorname{logs}$, approximately $z=0,0.5,1$, and 2 among 64 output times of the simulation. Then we analyze the alignments of dark-matter halo velocities to the local pancake plane in each catalog. Basically, to find a local pancake, we follow the methodology suggested by NL06.

As a first step in measurement, we select the halos which contain more than 50 particles (the left column of Table 1) since those halos in Millennium Run catalog which have too low particle numbers (less than 30) are poorly sampled and severely contaminated by the numerical noise. In other words, only those halos which have enough particles to define a halo density profile are reliable (V. Springel in private communication). Thus, we set the particle number cut-off at 50. Next, among the selected halos, we pick out field halos which are still in quasi-linear regime by using the method suggested by El-Ad \& Piran (1997) and Hoyle \& Vogeley (2002). The number of identified field halos is shown in the right column of Table 1. The field halos are suited for our purpose because they may not be seriously affected by gravitational forces of many other halos, retaining their initial conditions at the moment that they collapsed.

Using only those field halos, we determine local pancakes using the practical methods proposed by NL06. First, We find two nearest field halos for a field halo and define a local pancake plane enclosing all the three halos. Here, the local pancakes are found by changing the criterion distance $R_{c}$ from $0 h^{-1} \mathrm{Mpc}$ to $17 h^{-1} \mathrm{Mpc}$ which is a lower limit of $R_{1}$, the displacement vector to the first nearest halo. In other words, $R_{c}$ satisfies the following condition: $R_{c} \leq R_{1} \leq R_{2}$ where $R_{2}$ is the displacement vector to the second nearest neighbor.

With the determined local pancakes, then we calculate the alignment angles between a halo velocity vector and a vector normal to the local pancake plane, $\cos \theta$. Finally, we obtain the probability density distribution of $\cos \theta, p(\cos \theta)$, by counting the number of halos in each bin of $\cos \theta$. We note that in order to examine the dependence of the particle number cut-off which is used when we determine the halos, we recalculate the velocity-pancake alignment for those halos which include more than 30 particles though low-particle number halos suffer from simulation noise. From this calculation, we obtain almost perfectly consistent results with the results of the catalog in which halos include more than 50 particles as can be seen in Fig. 1 (particle number cut-off is 50) and Fig. 2 (particle number cut-off is 30). Thus, the velocity-pancake alignment is not affected by the choice of particle number cut-off.

Figure 1 shows $p(\cos \theta)$ with Poissonian error. The three columns correspond to three different cases of $z=0,1$, and 2 , respectively, and the three rows to three different scales of $R_{c}=0,3$, and 6 , respectively. The horizontal dotted line in each panel represents a random distribution. As can be seen in the right column, when $R_{c}$ is 0 , the halo velocity tends to be 
slightly aligned with the normal to the local pancake plane at $z=2$. This tendency becomes stronger as $R_{c}$ increases to $6 h^{-1} \mathrm{Mpc}$. It can be interpreted that the local pancaking effect arises at least around $z \simeq 2$. Meanwhile, at $z=1$, for the case of $R_{c}=0$, there is no alignment. Then local pancaking effect starts to be visible near $R_{c}=3 h^{-1} \mathrm{Mpc}$. Moreover, as one can see in the left column, when $R_{c}=0$ at $z=0$, the halo velocity does not tend to be aligned with the normal to the local pancake plane any more. Rather it tends to lie on the plane. The alignment signal begins to be seen when $R_{c}$ is approximately $6 h^{-1} \mathrm{Mpc}$. We infer from these phenomena that the pancaking effect disappears since the gravitational attraction forces from the close neighbor halos affect halo velocities rather than the initial pancaking effect. Therefore, we find the velocity-pancake alignments are strongest at $z \simeq 2$, indicating that the pancaking effect starts at $z \geqq 2$. We refer that we do not make more analysis of the velocity-pancake alignments for higher redshift to find a redshift at which pancaking effect starts since the number of halos decreases rapidly as redshift increases. Even if the value of $\eta$ itself might increase, the value of $\sigma_{\eta}$ which is inversely proportional to the number of halos would increase. Thus, it would be hard to determine precisely at which redshift both the values of $\eta$ and $\sigma_{\eta}$ reach maximums.

Figure 3 plots the average of $\cos \theta$ at four different $z=0,0.5,1$, and 2 as a function of linear characteristic pancake scale, $L_{p}$. We refer that $L_{p}$ is defined as the average distance between the center of mass (CM) for three halos on the local pancake plane to all the three halos, which was proposed by NL06. The horizontal dotted line in each panel represents $\langle\cos \theta\rangle$ of random distribution of the angles. As one can see in each panel, there is a specific range of $L_{p}$ where $\langle\cos \theta\rangle$ is relatively higher than those at the other pancake scales. This specific range implies local pancaking effect significantly occurs on specific pancake scale. Comparing to panels each other, overall $\langle\cos \theta\rangle$ increases, its slope is steeper and the specific range shifts to small $L_{p}$ when going to high redshift. This tendency can be clearly seen when we see that the regions of filled with oblique lines, which represent the area consisting of a few of the highest values, move to small $L_{p}$ as going to high redshift. More quantitatively, the peak value is approximately $5.035 \times 10^{-4}$ at $z=0$ while the one is $5.055 \times 10^{-4}$ at $z=2$. In addition, the peak position of histogram is approximately $2 h^{-1} \mathrm{Mpc}$ at $z=0$ whereas the one is $8 h^{-1} \mathrm{Mpc}$ at $z=2$. This gradual change with redshifts can be understood the same way as Fig. 1; after pancaking effect on halo velocities arises, it is gradually attenuated by gravitational force among halos, especially among very close halos. 


\section{THEORETICAL ANALYSIS}

In order to investigate the tendency of the anisotropic distribution of halo velocity theoretically, we construct an analytic model using the similar methodology to Lee \& Pen (2001). We begin on the Zel'dovich approximation (Zel'dovich 1970)

$$
\mathbf{x}=\mathbf{q}-D(t) \nabla \Psi(\mathbf{q}),
$$

which intrinsically estimates the formation of pancakes. Here, $\mathbf{x}$ is the Eulerian coordinate, $\mathbf{q}$ is the Lagrangian coordinate, $\Psi(\mathbf{q})$ is the linear velocity potential and $D(t)$ is the growth factor,

$$
D=\frac{5}{2} \Omega_{m} H(a) \int_{0}^{a} \frac{d a^{\prime}}{\left[a^{\prime} H\left(a^{\prime}\right)\right]^{3}} .
$$

By taking the time derivative of Zel'dovich approximation, we obtain equation of the velocity of cosmic particle smoothed on pancake scale,

$$
v_{i}=-\left(\dot{D} \partial_{i} \Psi+D \partial_{i} \dot{\Psi}\right) \propto-\left(\dot{D} q_{j} T_{i j}+D q_{j} \dot{T}_{i j}\right)
$$

Equation (3) is appropriate to investigate pancaking effect on halo velocity at early stage since equation (1) describes initial state of non-linear evolution. Also, we assume the density field is smoothed on the pancake scale, which is valid in this approximation because this approximation is available in the regime preceding the moment of the collapse to a pancake.

Using a similar logic given by Lee \& Pen (2001) who found an expression for the alignments between the tidal field and the halo position vectors, we take into account nonlinear effects into equation (3), and write an expression for the velocity alignments with the tidal field as

$$
\begin{aligned}
\left\langle v_{i} v_{j} \mid \mathbf{T}\right\rangle & =\frac{1-\eta}{3} \delta_{i j}+\eta K_{i j}, \\
K_{i j} & \equiv \dot{D}^{2} T_{i k} T_{k j}+2 D \dot{D} T_{i k} \dot{T}_{k j}+D^{2} \dot{T}_{i k} \dot{T}_{k j}
\end{aligned}
$$

where $\mathbf{T}$ and $\dot{\mathbf{T}}$ are tidal shear tensor and its time derivative, respectively. $\dot{D}$ is time derivative of $D$, which is

$$
\dot{D}=\frac{5}{2} \Omega_{m}\left[H^{\prime}(a) \dot{a} \int_{0}^{a} \frac{d a^{\prime}}{\left\{a^{\prime} H\left(a^{\prime}\right)\right\}^{3}}+\frac{\dot{a} H(a)}{a^{3} H(a)}\right],
$$

where $\quad H^{\prime}(a)=\frac{d H(a)}{d a}=-\frac{3}{2} \Omega_{m} a^{-4}\left(\Omega_{m} a^{-3}+\Omega_{\Lambda}\right)^{-1 / 2}, \quad \dot{a}=a H_{0}\left(\Omega_{m} a^{-3}+\Omega_{\Lambda}\right)^{1 / 2}$.

Notice that $\eta$ is a value in $[0,1]$. If $\eta$ is 1 , it represents a perfect correlation between local pancaking and halo velocity while if $\eta$ is 0 , it means there is no correlation between them. 
In velocity-velocity correlation, it is unlikely that the value of $\eta$ is close to unity since the velocity of a halo is not a conserved quantity and initial memory of local pancaking effect on velocity have been reduced. In order to show that the velocities of halos are not completely random but has some degree of alignments with the normal vectors to the local pancakes though The value of $\eta$ is expected to be very small, we have calculated the error on the value of $\eta$, that is, the standard deviation of $\eta$ for the case of no alignment. The formula for the error of $\eta, \sigma_{\eta}$ is given as $\left(4 / 15 N_{t o t}\right)^{1 / 2}$ where $N_{t o t}$ is the total number of halos (Lee \& Pen 2001). With defined error, if $\eta$ is larger than $3 \sigma_{\eta}$, one can say that there is a signal of true local pancaking effect. (1996)

We diagonalize equation (44) by applying the relation referred by Bond, Kofman, \& Pogosyan

$$
\dot{\lambda}_{i}=\frac{D \lambda_{i}}{1-D \lambda_{i}}
$$

where $\lambda_{i}$ and $\dot{\lambda}_{i}$ are an eigenvalue of the $\mathbf{T}$ and its time derivative, respectively. Thus, equation (15) is changed to

$$
K_{i i}=\frac{\lambda_{i}^{2}\left[\dot{D}\left(1-D \lambda_{i}\right)+D^{2}\right]^{2}}{\left(1-D \lambda_{i}\right)^{2}}, \quad K_{i j}=0(i \neq j)
$$

The eigenvalues $\lambda_{i}$ are ordered by $\lambda_{1}>\lambda_{2}>\lambda_{3}$.

To obtain the probability distribution, we assume probability density distribution of the alignment angles, $\cos \theta$, is Gaussian (Lee 2004)

$$
p(\hat{v} \mid \check{\mathbf{T}})=\frac{1}{\sqrt{(2 \pi)^{3} \operatorname{det}(M)}} \int_{0}^{\infty} \exp \left[-\frac{v_{i}^{T}\left(M^{-1}\right)_{i j} v_{j}}{2}\right] v^{2} d v
$$

where $M_{i j} \equiv\left\langle v_{i} v_{j} \mid \mathbf{T}\right\rangle$. Finally, we can express the probability distribution of $\cos \theta, p(\cos \theta)$ is,

$p(\cos \theta)=\frac{1}{2 \pi} \prod_{i=1}^{3}\left(1+\eta-3 \eta \check{\lambda}_{K i}\right)^{-\frac{1}{2}} \int_{0}^{2 \pi}\left(\frac{\sin ^{2} \theta \cos ^{2} \phi}{1+\eta-3 \eta \check{\lambda}_{K 1}}+\frac{\sin ^{2} \theta \sin ^{2} \phi}{1+\eta-3 \eta \check{\lambda}_{K 2}}+\frac{\cos ^{2} \theta}{1+\eta-3 \eta \check{\lambda}_{K 3}}\right)^{-\frac{3}{2}} d \phi$.

Here, $\check{\lambda}_{K i}=K_{i i} / \sum_{i} K_{i i}^{2}$.

To compare with the results from simulation analysis, we use $\Lambda$ CDM cosmological parameters, $h=0.73, \sigma_{8}=0.9, T_{c m b, 0}=2.725 \mathrm{~K}, \Omega_{b}=0.045, \Omega_{m}=0.25$, which are the same values as the parameters used in Millennium Simulation. First, we calculate $\sigma$ by using following equation:

$$
\sigma^{2}(R, z)=D(z)^{2} \int \frac{k^{2} d k}{2 \pi^{2}} P(k) T^{2}(k) W_{T H}^{2}(k R)
$$


where $D(z)$ is a growth function, $P(k)$ is primordial power spectrum that is $P(k) \propto k, T(k)$ is transfer function (Bardeen et al. 1986) and $W_{T H}$ is spherical top-hat window function. When evaluating equation (12), we use $L_{p}$ obtained by simulation analysis for $R$. Also, the redshift at which $L_{p}$ is determined in the simulation is chosen as $z$ value in equation (12).

Then we obtain $\lambda_{K i}$ in equation (10). We decide $\lambda_{K 1}$ as 1 because $\lambda_{1}=1$ means the formation of the structure like a pancake in the Zel'dovich approximation,

$$
\rho=\frac{\bar{\rho}}{\left(1-\lambda_{1}\right)\left(1-\lambda_{2}\right)\left(1-\lambda_{3}\right)},
$$

where $\bar{\rho}$ is the mean density of the universe and $\lambda_{1}, \lambda_{2}, \lambda_{3}\left(\lambda_{1}>\lambda_{2}>\lambda_{3}\right)$ are the eigenvalues of the local tidal field, T. For $\lambda_{K 2}$ and $\lambda_{K 3}$, we choose the most probable values of the following probability distribution,

$$
p\left(\lambda_{2}, \lambda_{3} \mid \lambda_{1}=1\right)=p\left(\lambda_{1}=1, \lambda_{2}, \lambda_{3}\right) / p\left(\lambda_{1}=1\right) .
$$

Here, $p\left(\lambda_{1}, \lambda_{2}, \lambda_{3}\right)$ and $p\left(\lambda_{1}\right)$ are the probability distribution derived by Doroshkevich (1970) and Lee \& Shandarin (1998):

$$
\begin{aligned}
p\left(\lambda_{1},\right. & \left.\lambda_{2}, \lambda_{3}\right)=\frac{3375}{8 \sqrt{5} \pi \sigma^{6}}\left(\lambda_{1}-\lambda_{2}\right)\left(\lambda_{2}-\lambda_{3}\right)\left(\lambda_{1}-\lambda_{3}\right) \exp \left(-\frac{3 I_{1}^{2}}{\sigma^{2}}+\frac{15 I_{2}}{2 \sigma^{2}}\right) \\
p\left(\lambda_{1}\right) & =\frac{\sqrt{5}}{12 \pi \sigma}\left[20 \frac{\lambda_{1}}{\sigma} \exp \left(-\frac{9 \lambda_{1}^{2}}{2 \sigma^{2}}\right)-\sqrt{2 \pi} \exp \left(-\frac{5 \lambda_{1}^{2}}{2 \sigma^{2}}\right) \operatorname{erf}\left(\sqrt{2} \frac{\lambda_{1}}{\sigma}\right)\left(1-20 \frac{\lambda_{1}^{2}}{\sigma^{2}}\right)\right. \\
& -\sqrt{2 \pi} \exp \left(-\frac{5 \lambda_{1}^{2}}{2 \sigma^{2}}\right)\left(1-20 \frac{\lambda_{1}^{2}}{\sigma^{2}}\right)+3 \sqrt{3 \pi} \exp \left(-\frac{15 \lambda_{1}^{2}}{4 \sigma^{2}}\right) \operatorname{erf}\left(\frac{\sqrt{3} \lambda_{1}}{2 \sigma}\right) \\
& \left.+3 \sqrt{3 \pi} \exp \left(-\frac{15 \lambda_{1}^{2}}{4 \sigma^{2}}\right)\right] .
\end{aligned}
$$

We put these eigenvalues in equation (91) to obtain the normalized eigenvalues, $\check{\lambda}_{K i}$.

Finally, we fit theoretical estimation of $p(\cos \theta)$, equation (11), to the numerical results as adjusting a correlation parameter $\eta$ in equation (11). We determine $\eta$ minimizing $\chi^{2}$ distribution

$$
\chi^{2}=\sum_{i} \frac{x_{s, i}-x_{t, i}}{\sigma_{s, i}}
$$

where $x_{s, i}$ and $x_{t, i}$ are the values from simulation analysis and from theory, respectively and $\sigma_{s, i}$ is the Poissonian error from simulation analysis. The comparison numerical results with theory is shown in Fig. 4. The filled circle points represent the numerically measured $p(\cos \theta)$ with Poissonian errors and solid line represents the analytic distribution. As can be seen in the figure, the simulation data points are in good agreement with our analytic formulation. 
Also, as shown in Table 2, the strength of local pancaking effect on the halo velocity depends on the redshift. At $z=2$, though halos are closely located at each other like $R_{c}=3 h^{-1} \mathrm{Mpc}$, $\eta$ is 0.011 , which turns out to be $15.3 \sigma_{\eta}$ (see Table 2). Thus, even though the value of $\eta$ is much less than unity, it is definitely a strong signal of velocity-pancake alignments, exceeding 15 times the standard deviation, $\sigma_{\eta}$. This strong signal which approximately amounts to $15 \sigma_{\eta}$ shows the existence of the velocity-pancake alignment. Even when $R_{c}=0$ at $z=2, \eta$ is 0.003 which is $4 \sigma_{\eta}$ which indicates that a pancaking effect appears even at $R_{c}=0$. Then going to present epoch, the effect is reduced by gravitational attraction. Finally, at $z=0$, when $R_{c}$ is $3 h^{-1} \mathrm{Mpc}, \eta$ is almost 0 and $\sigma_{\eta}$ is also 0 . In addition, as one can see the tendency in Fig. 4 and in Table 2, at $z=2$, pancaking effect becomes stronger as $R_{c}$ is approximately $5-6 h^{-1} \mathrm{Mpc}$ and then $\eta$ and $\sigma_{\eta}$ gradually decreases when $R_{c}$ is larger than $6 h^{-1} \mathrm{Mpc}$. In other words, the value of $\eta$ and $\sigma_{\eta}$ at $z=2$ is the largest with $R_{c} \simeq 6$. A similar tendency also appears at different redshifts, instead the range of $R_{c}$ is shifted to larger scale caused by the expansion of the universe. Thus, we analytically find that there is a specific range of pancake scale where pancaking significantly affects halo velocity. In addition, we show how the pancaking effect changes with different redshifts.

To find the tendency of the redshift dependence of $\eta$, we fit $\eta$ as a function of redshift to quadratic equation. The coefficients are shown in Table 3 . Figure 5 plots the quadratic fitting function (dashed line) and compares it with the simulation data points (filled circles). As Fig. 5 shows, we note that $\eta$ is a function of redshift, in a good agreement with the quadratic equation. Moreover, $\eta$ is a function of the characteristic pancake scale, $L_{p}$. We also fit $\eta\left(L_{p}\right)$ to quadratic equation which can be seen in Fig. 6. The dashed line and the filled circles represent the fitting function and the data points, respectively. We can see a good match between the quadratic functional form and the simulation data. The best-fit coefficients are shown in Table 4.

\section{DISCUSSION AND CONCLUSION}

We analyze the evolution of the pancaking effect by using millennium simulation. We obtain the signal of the strong alignment between the halo velocity and the normal to the local pancake plane at $z \simeq 2$ when characteristic pancake scale is approximately $3 h^{-1} \mathrm{Mpc}$. The alignment signal implies the first collapse of the protocloud may have occurred around $z \simeq 2$ and the size of the pancake may have been around $3 h^{-1} \mathrm{Mpc}$. This result is in agreement with Mo et al. (2005) which reports pancakes form at $z \simeq 2$ and their masses is approximately $5 \times 10^{12} \mathrm{M}_{\odot}$.

Then as shown in Fig. 1 and Fig. 3, the alignment signal evolves. Pancaking effect 
is weakened as going to low redshift, which means the pancaking effect on halo velocity is gradually attenuated by the gravitational attraction among the neighbor halos. In addition, pancake scale at low redshift is larger than that at high redshift.

We expect the local pancaking effect could be detected in observation. It may be possible to achieve the signal of the velocity alignment using the velocity data reconstructed from 2MASS redshift survey (Pirin et al. 2006). We intend to measure the signal in observation for our future project.

It is interesting to note that the pancakes are expected to have formed coincidentally at $z \simeq 2$ when the star formation rate in massive galaxies is the highest (Juneau et al 2006; Feulner et al. 2005), which is one of the phenomena referred to 'downsizing'. In 'downsizing' scenario, the stars in more massive galaxies formed at higher redshift and those in less massive galaxies recently formed, where 'antihierarchy' can be suggested. Recently, however, Neistein et al. (2006); Mouri \& Taniguchi (2006) show this phenomenon is natural in hierarchical clustering process. Nevertheless, a coincidence between the pancake formation epoch and the epoch of the heavily star-forming may imply the structure formation is not simply hierarchical but complicated (Cimatti et al. 2006).

In conclusion, we obtain the result that is the evolution of the velocity anisotropy induced by local pancake formation. This result would help to understand the unknowns in the galaxy formation.

The Millennium Run simulation used in this paper was carried out by the Virgo Supercomputing Consortium at the Computing Centre of the Max-Planck Society in Garching (http://www.map-garching.mpg.de/millennium). We are thankful to L. Gerard for the halo catalogs of the Millennium Run simulation and to V. Springel for useful discussion. We also appreciate the anonymous referee for helpful suggestions. This work is supported by the research grant No. R01-2005-000-10610-0 from the Basic Research Program of the Korea Science and Engineering Foundation. 


\section{REFERENCES}

Aragon-Calv, M. A. ,van de Weygaert, R. Jones, B. J. T., \& van der Hulst, J. M. 2007, ApJ, in press (astro-ph/0610249)

Bardeen, J.M., Bond, J.R., Kaiser, N., \& Szalay, A.S. 1986, ApJ, 304, 15

Bond, J., R., Kofman, L., \& Pogosyan, D. 1996, Nature, 380, 603

Brunino, R., Trujillo, I., Pearce, F. R., \& Thomas, P. A. 2007, MNRAS, in press (astro-ph/0609629)

Cimatti, A., Daddi, E., \& Renzini, A. 2006, A\&A, 453, 29

Croton, D. J., Springel, V., White, S. D. M., De Lucia, G., Frenk, C. S., Gao, L., Jenkins, A., Kauffmann, G., Navarro, J. F., \& Yoshida, N. 2006, MNRAS, 367, 864

Doroshkevich, A. G. 1970, Astrofizika, 3, 175

El-Ad, H., \& Piran, T. 1997, ApJ, 491, 421

Feulner, G., Gabasch, A., Salvato, M., Drory, N., Hopp, U., \& Bender, R. 2005, ApJ, 633, L9

Glazebrook, K. et al. 2005, Nature, 430, 181

Hahn, O., Porciani, C., Carollo, M. C., \& Dekel A. 2007, MNRAS, 375, 489

Hoyle, F., \& Vogeley, M. S. 2002, ApJ, 566, 641

Juneau, S. et al. 2005, ApJ, 619L, 135

Lee, J. \& Shandarin, S. F. 1998, ApJ, 500, 14L

Lee, J., \& Pen, U. L. 2001, ApJ, 555, 106

Lee, J., \& Pen, U. L. 2002, ApJ, 567, L111

Lee, J. 2004, ApJ, 614, L1

Mo, H. J., Yang, X., van den Bosch, F. C., \& Katz, N. 2005, MNRAS, 363, 1155

Mouri, H., \& Taniguchi, Y. 2006 A\&A, 459, 371

Noh, Y., \& Lee, J. 2006, ApJ, 652, L71 
Neistein, E., van den Bosch, F. C., \& Dekel, A. 2006, ApJ, 372, 933

Erdogdu, P., Lahav, O., Huchra, J. P., Colless, M., Cutri, R. M., Falco, E., George, T., Jarrett, T., Jones, D. H., Macri, L. M., Mader, J., Martimbeau, N., Pahre, M. A., Parker, Q. A., Rassat, A., \& Saunders, W. 2006, MNRAS, 373, 45

Shandarin, S. F., Melott, A. L., McDavitt, K., Pauls, J. L., \& Tinker, J. 1995, Phys. Rev. Lett., 75,7

Spergel, D. N. et al. 2003, ApJ, 148, 175

Springel, V. et al. 2005, Nature, 435, 629

Zel'dovich, Ya. B. 1970, A\&A, 5, 84 


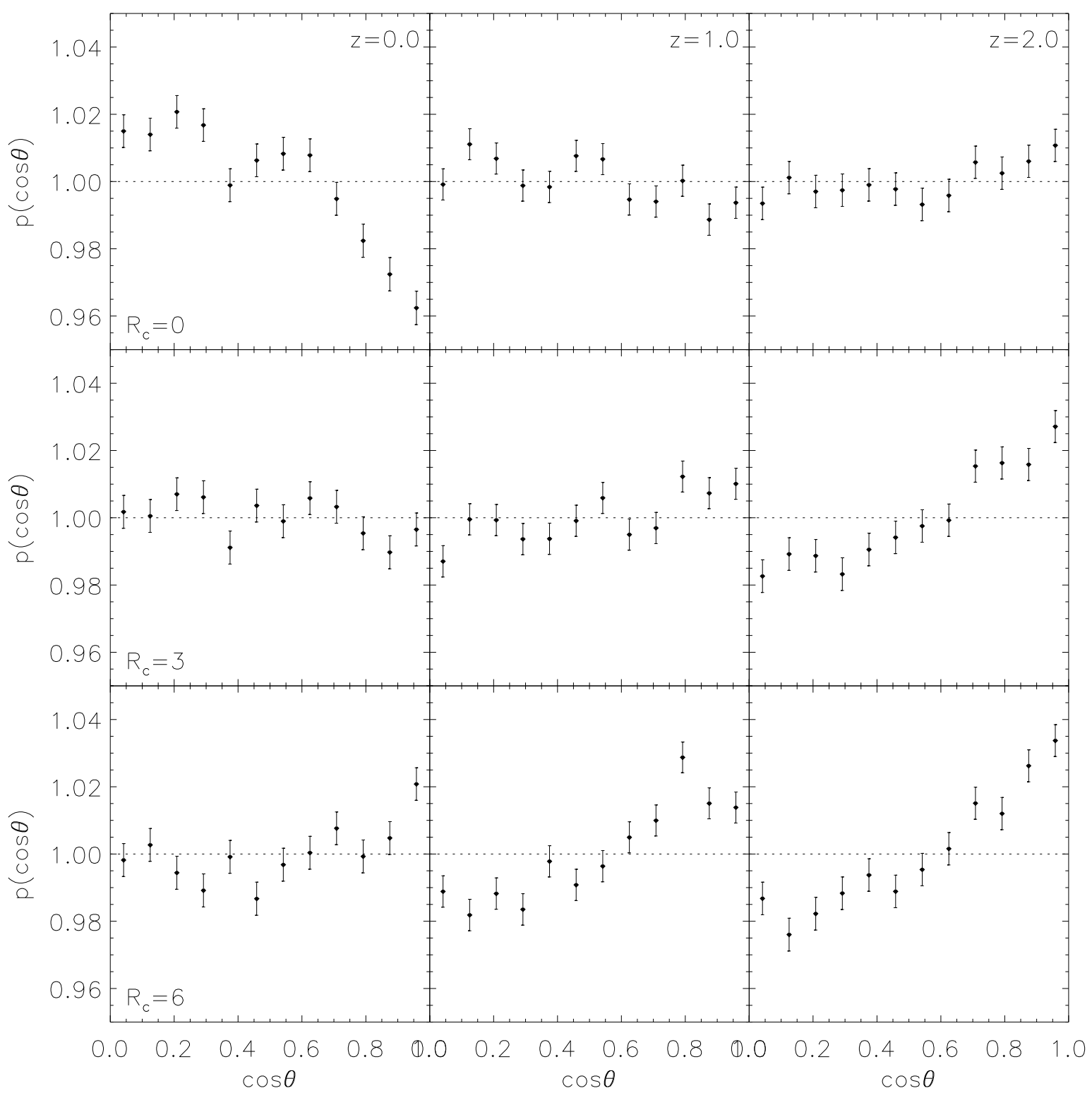

Fig. 1.- Probability density distributions of the cosines of the relative angles between the field halo velocities and the directions normal to the local planes enclosing the two neighbor field halos for the cases of the distance threshold: $R_{c}=0,3,6 h^{-1} \mathrm{Mpc}$ at different redshift: $z=0,1,2$ from Millennium Run simulation. The errors are Poissonian and the horizontal dotted lines represent no alignment. Note that halos which contain more than 50 particles are determined 


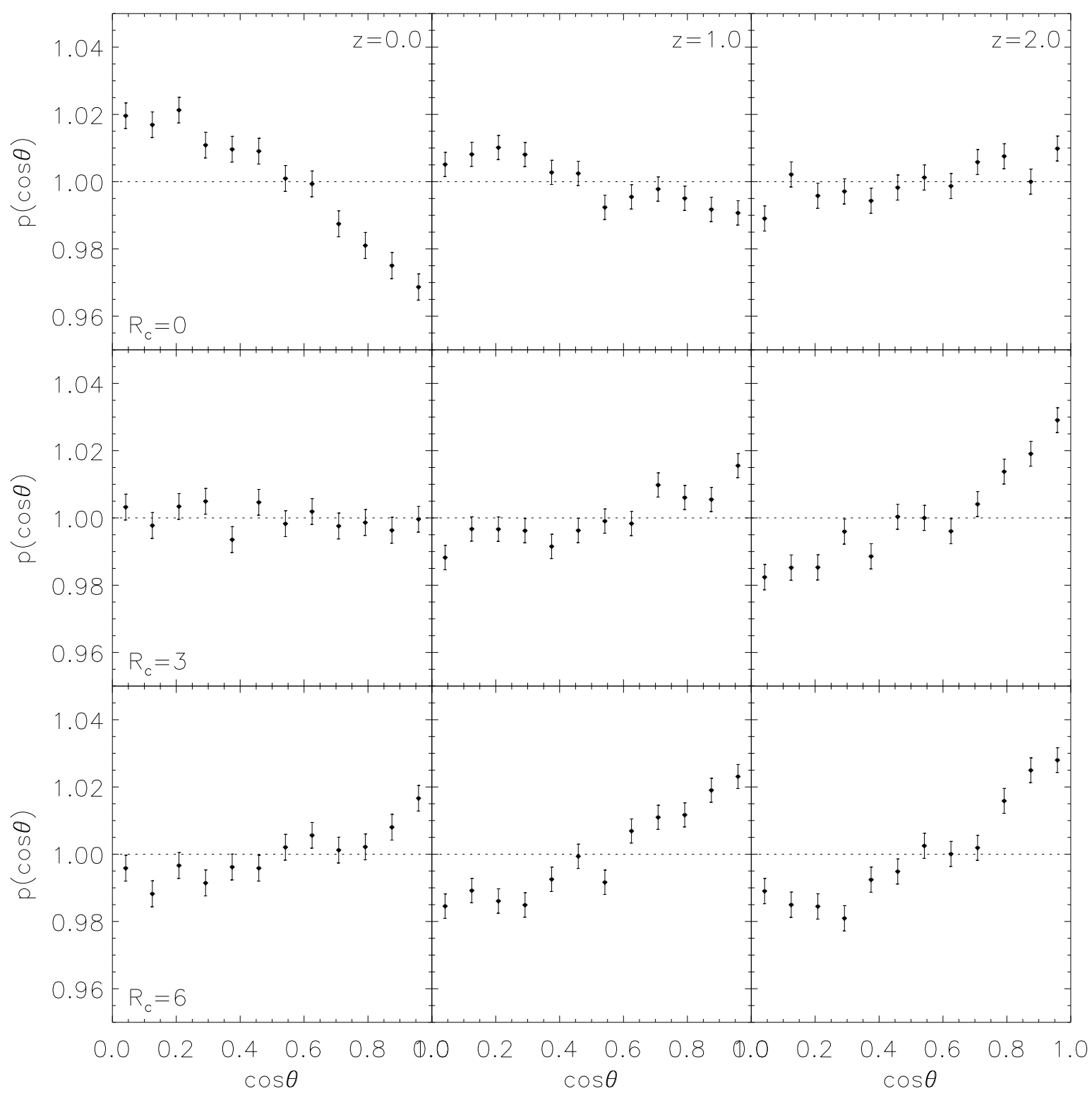

Fig. 2.- The same figure as Fig. 1 but using the halos which contain more than 30 particles. 


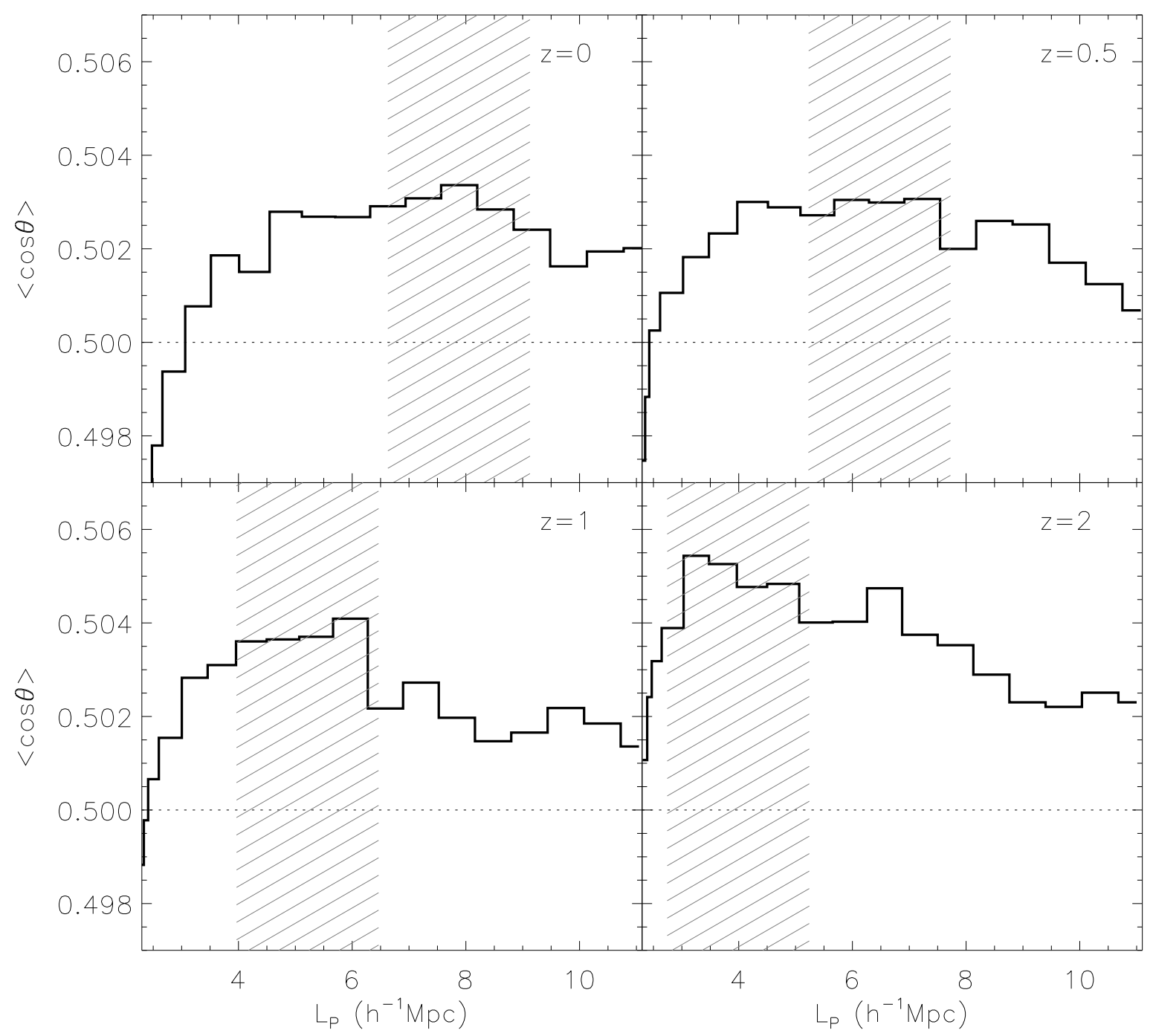

Fig. 3.- The average of the cosines angles $(\cos \theta)$ between the halo velocities and the local pancake planes whose definition is the same as Fig. 1, corresponding to the change of characteristic linear size of the pancake, $L_{p}$ at four different redshift: $z=0,0.5,1,2$. The horizontal dotted lines represent no correlation. 


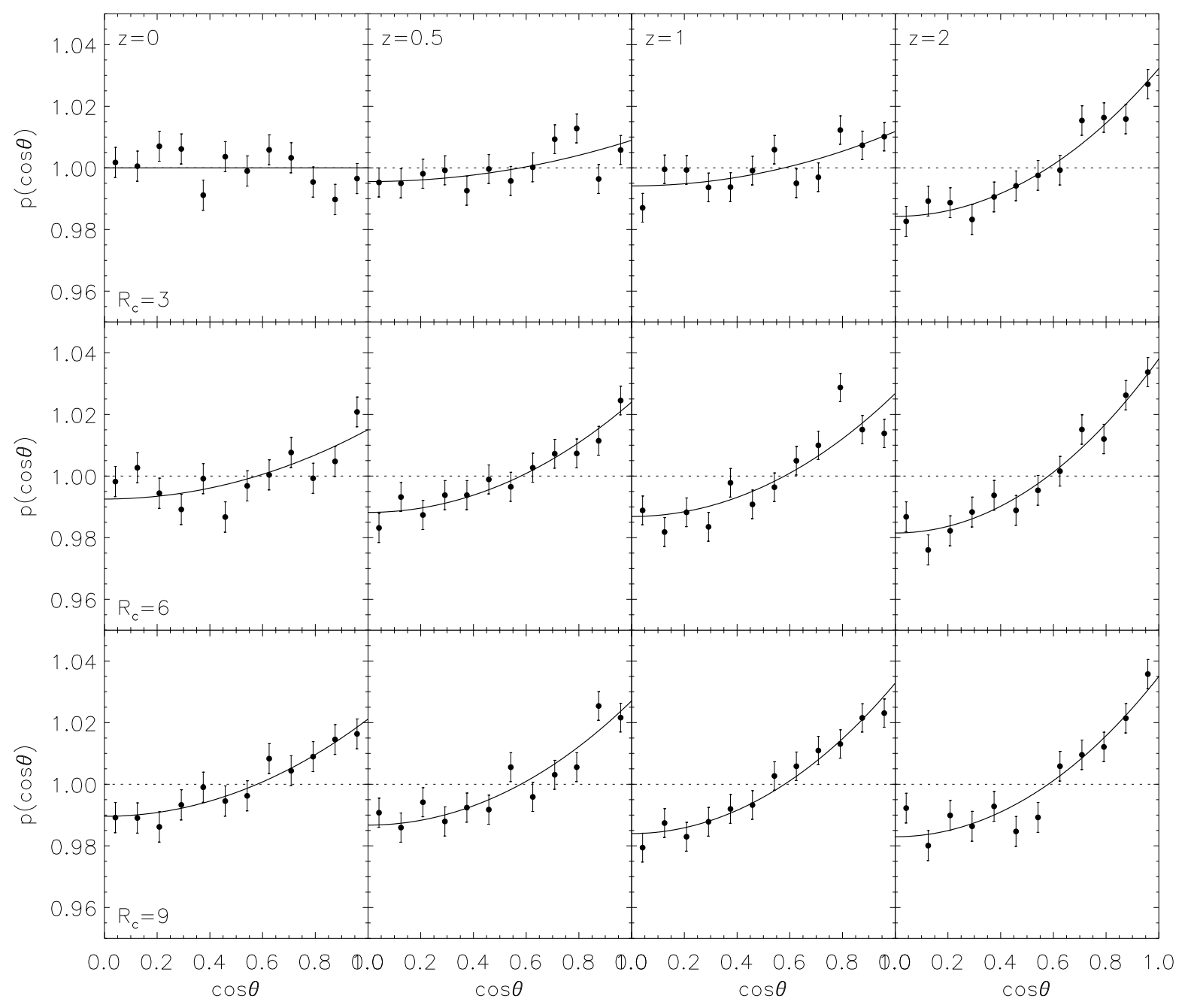

Fig. 4.- Probability density distributions of the $\cos \theta$ s. Filed circle with Poissonian errors represent the results from the simulation while solid line stand for the analytic prediction with correlation parameter $\eta$. The horizontal dotted lines correspond to no correlation. 


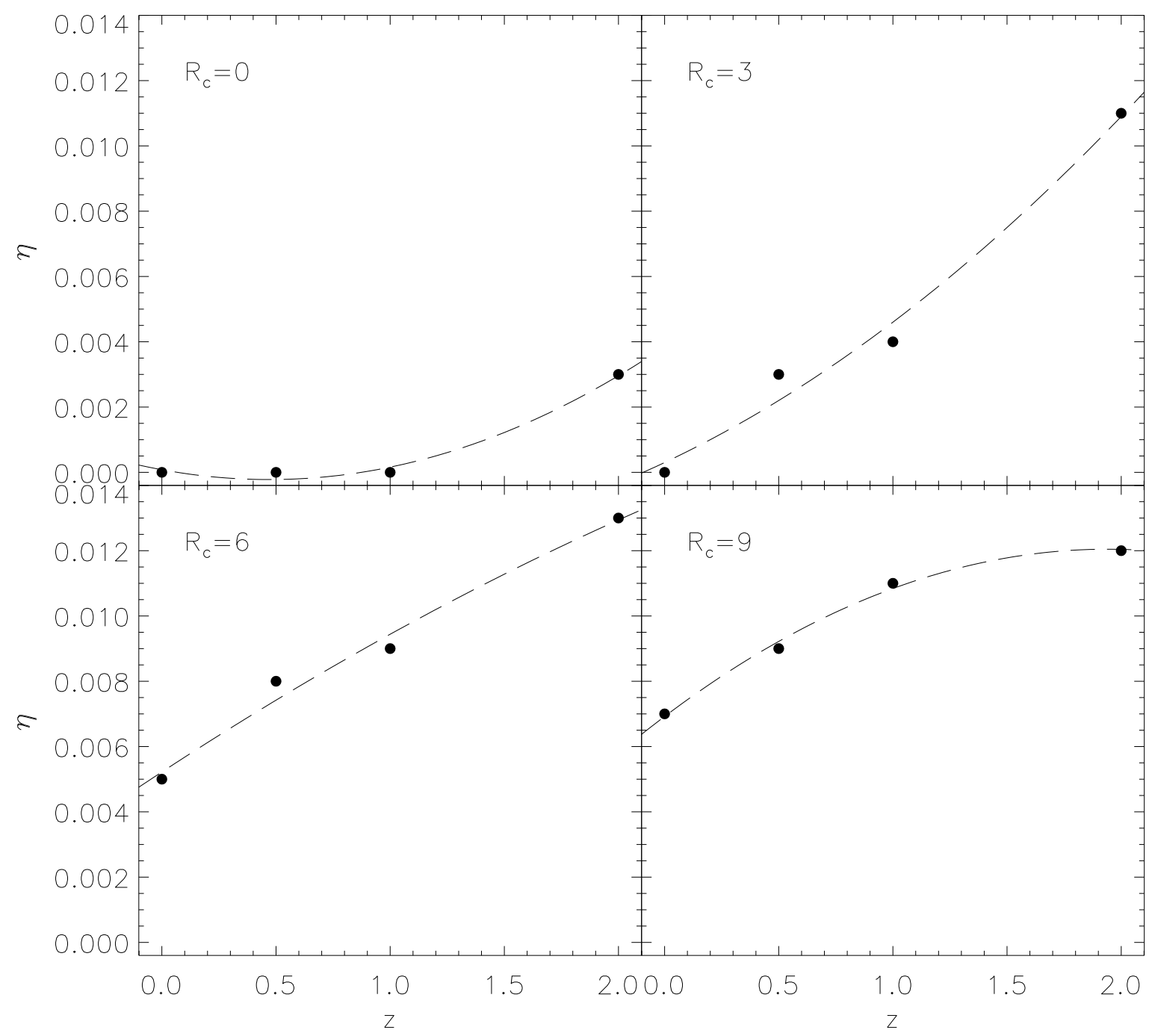

Fig. 5. $-\eta$ as a function of redshifts. Filed circle represents the numerical measurement in Millennium Simulation. Dashed line is a quadratic fitting function at different $R_{c}$ scale. 


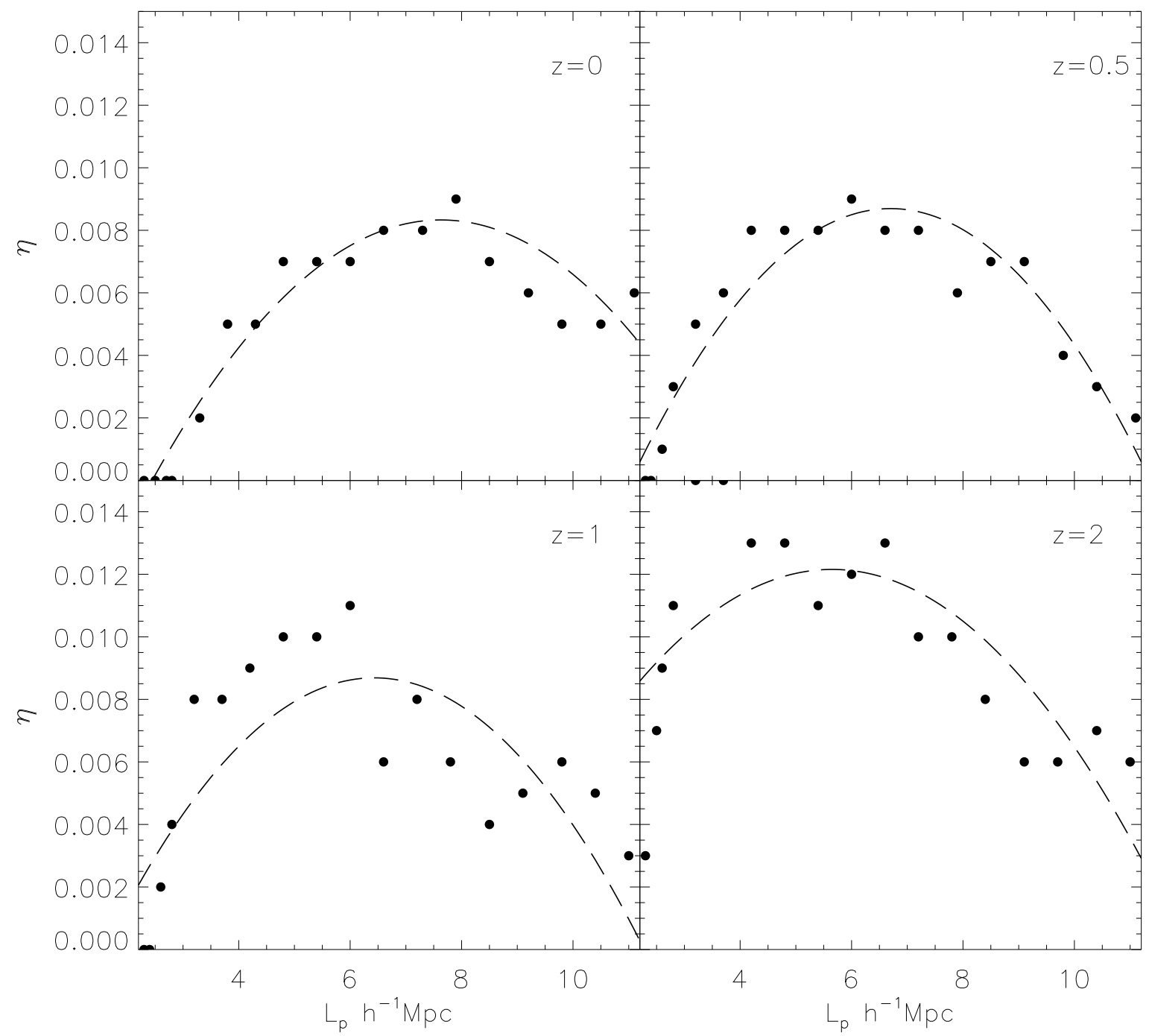

Fig. 6.- The same as Fig. 5 but as a function of characteristic pancake scale, $L_{p}$. 
Table 1. left: Total number of identified halos whose contain more than 50 particles in each redshift catalog, right: The number of field halos

\begin{tabular}{|c|c|c|}
\hline$z$ & $N_{\text {total }}$ & $N_{\text {field }} \quad\left(\frac{N_{\text {field }}}{N_{\text {total }}} \%\right)$ \\
\hline 0 & 6126689 & $502108(8.2)$ \\
\hline 0.5 & 6631145 & $542554(8.2)$ \\
\hline 1 & 6817225 & $558138(8.2)$ \\
\hline 2 & 6222187 & $516774(8.3)$ \\
\hline
\end{tabular}


Table 2. Correlation parameter $\eta$ corresponding to $R_{c}$ and redshift, $z$ with its standard deviation for the case of no alignment. The formula for the error of $\eta, \sigma_{\eta}$ is given as $\left(4 / 15 N_{t o t}\right)^{1 / 2}$ where $N_{\text {tot }}$ is the total number of halos (Lee \& Pen 2001).

\begin{tabular}{ccccc}
\hline \hline \multirow{2}{*}{$\begin{array}{c}R_{c} \\
\left(h^{-1} \mathrm{Mpc}\right)\end{array}$} & 0 & \multicolumn{4}{c}{$\mathrm{z}$} \\
\cline { 2 - 5 } & 0.5 & 1 & 2 \\
\hline 3 & $0.000\left(0.0 \sigma_{\eta}\right)$ & $0.003\left(4.3 \sigma_{\eta}\right)$ & $0.004\left(5.8 \sigma_{\eta}\right)$ & $0.011\left(15.3 \sigma_{\eta}\right)$ \\
6 & $0.005\left(6.9 \sigma_{\eta}\right)$ & $0.008\left(11.4 \sigma_{\eta}\right)$ & $0.009\left(13.0 \sigma_{\eta}\right)$ & $0.013\left(18.1 \sigma_{\eta}\right)$ \\
9 & $0.007\left(9.6 \sigma_{\eta}\right)$ & $0.009\left(12.8 \sigma_{\eta}\right)$ & $0.011\left(15.9 \sigma_{\eta}\right)$ & $0.012\left(16.7 \sigma_{\eta}\right)$ \\
\hline
\end{tabular}


Table 3. Fitting parameters for $\eta$ as a function of redshift, $z$. Fitting functions are quadratic function, $\eta(z)=a z^{2}+b z+c,\left(10^{-3}\right)$

\begin{tabular}{cccc}
\hline \hline$z$ & $a$ & $b$ & $c$ \\
\hline 0 & 1.36 & -1.28 & 0.08 \\
0.5 & 1.00 & 3.30 & 0.30 \\
1 & -0.36 & 4.58 & 5.22 \\
2 & -1.36 & 5.28 & 6.92 \\
\hline
\end{tabular}


Table 4. The same as Table 3 but as a function of linear characteristic pancake scale, $L_{p}$, $\eta\left(L_{p}\right)=a L_{p}^{2}+b L_{p}+c,\left(10^{-3}\right)$

\begin{tabular}{cccc}
\hline \hline$z$ & $a$ & $b$ & $c$ \\
\hline 0 & -0.31 & 4.73 & -9.71 \\
0.5 & -0.40 & 5.36 & -9.26 \\
1 & -0.37 & 4.76 & -6.62 \\
2 & -0.30 & 3.39 & 2.58 \\
\hline
\end{tabular}

